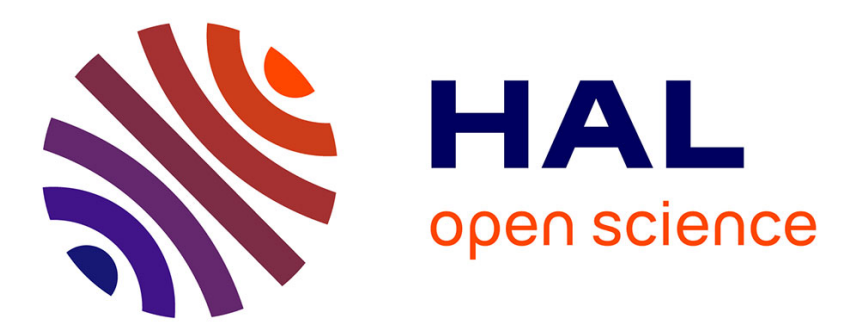

\title{
How plants connect pollination and herbivory networks and their contribution to community stability
}

\author{
Alix Marianne Carine Sauve, Elisa Thébault, Michael J. O. Pocock, Colin
} Fontaine

\section{- To cite this version:}

Alix Marianne Carine Sauve, Elisa Thébault, Michael J. O. Pocock, Colin Fontaine. How plants connect pollination and herbivory networks and their contribution to community stability. Ecology, 2015, 9 (4), pp.908-917. 10.1890/15-0132.1 . hal-01301160

\section{HAL Id: hal-01301160 https://hal.sorbonne-universite.fr/hal-01301160}

Submitted on 11 Apr 2016

HAL is a multi-disciplinary open access archive for the deposit and dissemination of scientific research documents, whether they are published or not. The documents may come from teaching and research institutions in France or abroad, or from public or private research centers.
L'archive ouverte pluridisciplinaire HAL, est destinée au dépôt et à la diffusion de documents scientifiques de niveau recherche, publiés ou non, émanant des établissements d'enseignement et de recherche français ou étrangers, des laboratoires publics ou privés. 


\title{
How plants connect pollination and herbivory networks and their contribution to community stability
}

\author{
Alix M. C. Sauve, ${ }^{1,2,3,4,6}$ Elisa Thébault,${ }^{3}$ Michael J. O. Pocock ${ }^{5}$ and Colin Fontaine ${ }^{4}$ \\ ${ }^{1}$ Department of Computer Science, University of Bristol, Merchant Venturers' Building, Woodland Road, Bristol, BS8 1UB, \\ United Kingdom \\ ${ }^{2}$ School of Biological Sciences, University of Bristol, Bristol Life Sciences Building, 24 Tyndall Avenue, Bristol, BS8 1TQ, \\ United Kingdom \\ ${ }^{3}$ Institute of Ecology and Environmental Sciences - Paris, UMR 7618 (CNRS, UPMC, ENS, IRD, AgroParisTech), \\ Université Pierre et Marie Curie, 7 quai Saint Bernard, Paris, 75005, France \\ ${ }^{4}$ Centre d'Ecologie et des Sciences de la Conservation (CESCO UMR7204), Sorbonne Universités, MNHN, CNRS, UPMC, CP51, \\ 55 rue Buffon, Paris, 75005, France \\ ${ }^{5}$ Centre for Ecology and Hydrology, Crowmarsh Gifford, Wallingford, Oxfordshire, OX10 8BB, United Kingdom
}

Abstract. Pollination and herbivory networks have mainly been studied separately, highlighting their distinct structural characteristics and the related processes and dynamics. However, most plants interact with both pollinators and herbivores, and there is evidence that both types of interaction affect each other. Here we investigated the way plants connect these mutualistic and antagonistic networks together, and the consequences for community stability. Using an empirical data set, we show that the way plants connect pollination and herbivory networks is not random and promotes community stability. Analyses of the structure of binary and quantitative networks show different results: the plants' generalism with regard to pollinators is positively correlated to their generalism with regard to herbivores when considering binary interactions, but not when considering quantitative interactions. We also show that plants that share the same pollinators do not share the same herbivores. However, the way plants connect pollination and herbivory networks promotes stability for both binary and quantitative networks. Our results highlight the relevance of considering the diversity of interaction types in ecological communities, and stress the need to better quantify the costs and benefits of interactions, as well as to develop new metrics characterizing the way different interaction types are combined within ecological networks.

Key words: antagonism; community stability; herbivory network; multiple interaction types; mutualism; network structure; pollination network.

\section{INTRODUCTION}

Plants are the basis of ecosystems: many species and interactions depend on the composition of plant communities, the diversity of their components and their abundances (Maron and Crone 2006). In terrestrial ecosystems, two biotic interactions with animals strongly impact upon plant fitness: animal pollination and herbivory. Animal pollination is involved in the sexual reproduction of $85 \%$ of angiosperm species, representing $80 \%$ of all terrestrial plants (Ollerton et al. 2011), while $18 \%$ of plant biomass is consumed by herbivores (McCall and Irwin 2006). Therefore, plants are connected to animals through both mutualistic and antagonistic interactions, e.g., pollination and herbivory, respectively.

Manuscript received 23 January 2015; revised 21 September 2015; accepted 28 September 2015. Corresponding Editor: N. M. Williams.

${ }^{6}$ E-mail: alix.sauve@cri-paris.org
At the community level, the way plants are connected to each of these animal communities has been studied by applying food web approaches, so providing a unified framework for analyzing plant and animal communities (Memmott 1999, Van Veen et al. 2008). Such network approaches have produced findings that enlighten our understanding of the structure of plantanimal communities (Lewinsohn et al. 2006). In particular, comparative studies have shown that mutualistic and antagonistic networks between plants and animals tend to exhibit different architectures, the former being more nested than the latter, but the latter appearing more modular (Bascompte et al. 2003, Thébault and Fontaine 2008, 2010, Genini et al. 2010, Fontaine et al. 2011). More interestingly, the specific patterns of both mutualistic and antagonistic networks have been shown to be related to their stability and response to perturbation (Okuyama and Holland 2008, Thébault and Fontaine 2010, Allesina and Tang 2012). However, these lines of research have mainly considered mutualistic and antagonistic networks separately, and studies 
combining both types of interaction within a single framework have questioned these findings and raised new issues (Ings et al. 2009, Fontaine et al. 2011, Sauve et al. 2014).

One of these issues is that the interactions of plant species with pollinators and herbivores are not independent from each other. Herbivory can reduce pollination by altering floral display (Strauss 1997), by activating chemical defenses of plants (Adler et al. 2006), and by decreasing availability and quality of rewards for pollinators (Strauss 1997, Lehtilä and Strauss 1999, Adler et al. 2006). Reciprocally, several floral traits involved in pollinator attraction have been shown to attract herbivores as well. This is the case for corolla size and color (Strauss et al. 2002), nectar (Adler and Bronstein 2004), and floral scent (Theis 2006). Hence, we could improve our understanding of the dynamics of plant-pollinator communities by taking into account herbivores, and vice versa.

Another issue is that network architecture strongly influences how perturbations spread from species to species within communities (Tylianakis et al. 2010), but such an effect can change whether we consider pollination and herbivory networks in isolation or connected in a single network. For example, nested and modular network structures strongly affect the stability of pollination and herbivory communities in isolation (Thébault and Fontaine 2010) but not when these networks are connected (Sauve et al. 2014). However, even Sauve et al. (2014) still considered the structures of mutualistic and antagonistic networks separately and thus ignored how the different types of interactions are combined.

Here we aimed to improve our understanding of (1) the way plants connect pollination and herbivory networks and (2) the consequences of this connection pattern for community stability. To do so, we analysed one of the very few highly resolved data sets simultaneously describing pollination and herbivory interactions (Pocock et al. 2012a,b). Our hypotheses were that plants that are generalists in the pollination networks are also generalists in the herbivory networks, because animals may respond similarly to plant signals (e.g., Strauss et al. 2002, Adler and Bronstein 2004, Theis 2006). In addition, we expected that plants that share the same pollinators might share the same herbivores, since interaction conservatism of plants has been found in both pollination and herbivory networks, meaning that phylogenetically related plants tend to share the same interaction partners (Ives and Godfray 2006, Rezende et al. 2007, Elias et al. 2013, Rafferty and Ives 2013). Finally, the way plants connect pollination and herbivory networks should affect the spread of perturbations from one network to the other (Fontaine et al. 2011). We thus hypothesized that the observed structural pattern of interconnected networks should affect community stability.

\section{Methods}

The data set: a quantitative network combining different types of interactions

To test our hypothesis, we analysed the interaction network of the Norwood farm sampled over 2007 and 2008 in the Somerset, UK (Pocock et al. 2012a,b; data available online). ${ }^{7}$ It is one of the few networks based on field sampling that integrates various interaction types with such taxonomic resolution. The network is also quantitative, that is the frequency of interactions between species was recorded during sampling or was estimated. For our analysis, we considered a subset of the original network: the plants and their direct interaction partners. For the mutualistic partners, this includes all flower visitors, hereafter referred to as "pollinators." For the antagonistic partners, this includes seed-feeding insects, rodents, birds, and aphids, hereafter referred to as "herbivores." As network topology (presence/ absence of interactions) and the distribution of interaction strengths both shape network architecture and can affect community stability, we first analysed a binary version of the network and, second, repeated our analysis using the quantitative version of the network.

We defined the binary interaction matrix $\boldsymbol{T o b s}=(\boldsymbol{t}(\mathbf{o b s}, \boldsymbol{i}, \boldsymbol{j}))$ of the whole network $\left(i \in \llbracket 1, S_{\text {animal }} \rrbracket\right.$ and $\left.j \in \llbracket 1, S_{\text {plant }} \rrbracket\right)$, such that $t_{(\mathrm{obs}, i j)}=1$ if plant species $j$ and animal species $i$ interact and $t_{(\mathrm{obs}, i j)}=0$ otherwise. $\mathbf{T}_{\text {obs }}$ exhibits two partitions: a matrix $\mathbf{M}_{\text {obs }}$ for the mutualistic network (dimensions are $\left\{S_{\text {poll }}, S_{\text {plant }}\right\}$ ), and a matrix $\mathbf{A}_{\text {obs }}$ for the antagonistic network (dimensions are $\left.\left\{S_{\text {herb }}, S_{\text {plant }}\right\}\right)$, so that Tobs $=\left[\begin{array}{c}\text { Mobs } \\ \boldsymbol{A o b s}\end{array}\right]$. We also defined a valuation function $q$ such that $q(\boldsymbol{T o b s})=\left[\begin{array}{c}q(\text { Mobs }) \\ q(\boldsymbol{A o b s})\end{array}\right]$ is the quantitative network whose elements $q\left(t_{(\mathrm{obs}, i j)}\right)$ correspond to the interaction frequencies between plant species $j$ and animal species $i$.

\section{Quantifying interactions for different interaction types}

Quantifying interactions in a network made of different interaction types is not an easy task because we need to find a common currency for interaction strengths (Fontaine et al. 2011, Kéfi et al. 2012, 2015). Studies focusing on one interaction type generally use interaction frequencies to investigate the structure of quantitative networks (Vazquez et al. 2005). However, observed interaction frequencies can vary a lot among interaction types. For example, in our data set, the differences in frequencies are up to 15 orders of magnitude within antagonistic networks (comparing plant-aphid with plant-seed predator interactions) and 12 orders of magnitude between network types (comparing plant-aphid with plant-flower visitor interactions). Such differences in interaction frequencies arise from the differences in

\footnotetext{
${ }^{7}$ http://dx.doi.org/10.5061/dryad.3s36r118
} 
population abundances among groups (aphids are much more numerous that seed-eating birds for instance). Thus, the use of interaction frequencies as a common currency for interaction strengths is not appropriate. To overcome this problem, we estimated the interaction preferences $\gamma_{i j}$ from the interaction frequencies given by $q$ (Tobs) following the method of Staniczenko et al. (2013). The preference $\gamma_{i j}$ corresponds to the interaction rate of an individual of a species $i$ with its interacting partner $j$ corrected for the variation in species $j$ abundance.

\section{Metrics describing the interconnection patterns between the different types of networks}

We used two metrics to characterize the way pollination and herbivory networks are interconnected by the plant guild: the correlation between the mutualistic and antagonistic generalism of plants, and the correlation between the similarities of pollinators and herbivores among plant pairs. Here, we provide a description of these metrics both for binary and quantitative networks. As all plants are not necessarily pollinated and consumed by animals, our metrics for interconnection patterns focus on the plants that interact both with pollinators and herbivores. We define them as interconnecting plants.

Correlation between plant generalism in mutualistic and antagonistic networks. - To investigate whether plants that tend to be generalists in one network are generalists in the other network (Fig. 1a), we first evaluated plant generalism in each sub-network and then calculated a correlation coefficient between the two. We used a Kendall rank coefficient of correlation $\tau$, which is nonparametric, to assess the correlation between plant mutualistic and antagonistic generalism because the relationship between plant mutualistic and antagonistic generalism was not linear. For the binary network, plant generalism was characterized by their degrees: the mutualistic degree $d_{\mathrm{M}}$ (the number of pollinator species that pollinate a given plant species) and the antagonistic degree $d_{\mathrm{A}}$ (the number of herbivore species that consume a given plant species) of plants. For the quantitative network, we calculated the Shannon diversity of preferences $\gamma_{i j}$ for each plant species $i$ to estimate the generalism of plant species in each type of networks (Bersier et al. 2002, Blüthgen et al. 2008). It is written as $H_{i}=-\sum_{j \in V} \frac{\gamma_{j i}}{\Gamma_{i}} \ln \left(\frac{\gamma_{j i}}{\Gamma_{i}}\right)$ where $\Gamma_{i}=\sum_{j \in V} \gamma_{j i}$ and $V$ is the set of animal species considered (either pollinators or herbivores).

Correlation between similarities of plant interaction partners in mutualistic and antagonistic networks. - To evaluate whether plants that share pollinators also share herbivores, we first computed the interaction overlap among each pair of plant species, in each network. When considering the binary network, we computed the Jaccard similarity coefficient between plant pairs, defined as the number of shared interaction partners divided by the total number of a

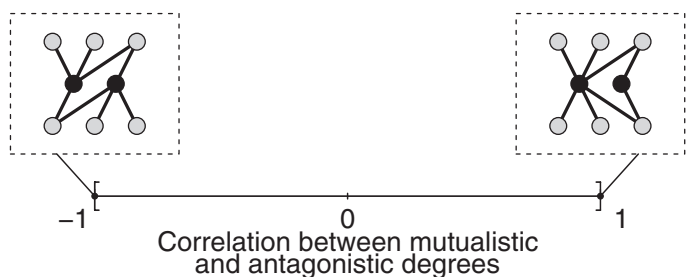

b

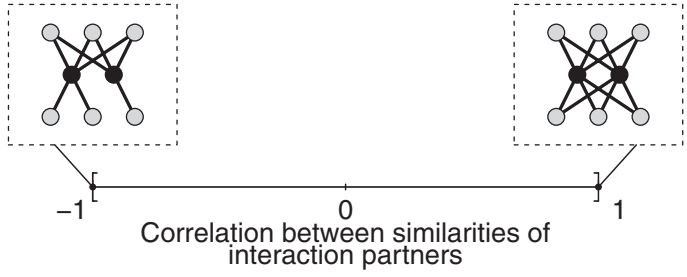

FIG. 1. Network representations of interconnected networks showing strong negative and strong positive correlations (a) between the mutualistic degree $\left(d_{\mathrm{M}}\right.$; the number of pollinator species that pollinate a given plant species) and the antagonistic degree $\left(d_{\mathrm{A}}\right.$; the number of herbivore species that consume a given plant species) of plants and (b) between the similarities of interaction partners among plants between pollination and herbivory networks. These metrics of interconnection patterns focus on black nodes (plants).

interaction partners involved (i.e., the maximum number of interactions they could share). When considering the quantitative network, we calculated the quantitative similarities of preferences $\gamma_{i j}$ of all pairs of plants in each type of network using the generalized Jaccard coefficient. The similarity between the sets of interaction partners of type $V$ (pollinators or herbivores) of plants species $x$ and $y$ is defined as $\frac{\sum_{i \in V} \min \left(\gamma_{i x}, \gamma_{i y}\right)}{\sum_{i \in V} \max \left(\gamma_{i x}, \gamma_{i y}\right)}$. We then calculated the Kendall $\tau$ between the Jaccard coefficients in mutualistic and antagonistic networks across all pairs of plants (Szell et al. 2010). A correlation close to 1 reflects a network in which pairs of plants that share the same pollinators tend to share the same herbivores. A correlation close to -1 reflects a network in which plants that share the same pollinators do not share the same herbivores and vice versa (Fig. 1b).

\section{Testing for the contribution of plant densities to interconnection patterns}

The Leaf Area Index (LAI) and the number of floral units (FU) can both be considered as proxies for plant species densities and resource abundances for either herbivores or pollinators. To test whether plant densities contribute to the correlation between mutualistic and antagonistic degrees of plants, we performed partial correlations, with the Kendall method, between mutualistic and antagonistic degrees, with either the LAI or the number of floral units (FU) held constant $(r(d \mathrm{M}, d \mathrm{~A})$. LAI, $r(d \mathrm{M}, d \mathrm{~A}) . \mathrm{FU}$ respectively). This allowed us to test whether the observed correlation between plant mutualistic and antagonistic degrees can be explained by differences in plant densities. Most abundant plants are expected to attract more pollinators and herbivores than less 
abundant ones, and to be as well more frequently sampled by observers, leading to higher observed values of degree (Blüthgen et al. 2008). The LAI and the number of floral units per plant species were measured on the sampled transects and scaled to the whole farm area (Pocock et al. 2010, Evans et al. 2015). Floral units were determined by the size and arrangement of flowers according to Dicks et al. (2002): they were such that a middle-sized bee would tend to fly, rather than walk, from one floral unit to another.

\section{Community stability}

Following classical theoretical studies on ecological network stability (May 1972, Neutel et al. 2002, 2007, Allesina and Tang 2012), we considered community matrices to investigate the relationship between interconnection patterns and community stability. A community matrix $\mathbf{J}$ is the Jacobian matrix of a population dynamics model at equilibrium with all species present. Each element $J_{i j}(\{i, j\} \in \mathbb{I}$ $\left.1, S_{\text {plant }}+S_{\text {animal }} \rrbracket\right)$ quantifies the effect that species $j$ has on species $i$ growth rate. In the case of an antagonistic interaction (i.e., herbivory) with species $i$ consuming species $j$, we have $J_{i j}>0$ and $J_{j i}<0$. In the case of a mutualistic interaction (i.e., pollination) between species $i$ and $j$, we have $J_{i j}>0$ and $J_{j i}>0$.

Following Neutel et al., we evaluated stability as the minimum intraspecific competition (i.e., minimum self-damping) necessary to community stability (Neutel et al. 2002, 2007). In other words, $J_{i i}=-s$ and we looked for the minimum value of $s$ such that all eigenvalues have negative real parts (see Appendix S1).

To examine both the effect of network topology (presence/absence of interactions) and the distribution of interaction strengths on community stability, we estimated the elements of the Jacobian matrix $J_{i j}$ in two ways. First, to investigate the effect of network topology on community stability, we drew all the off-diagonal non-zero elements of $\mathbf{J}$ from a half-normal distribution $\left|N\left(\mu, \sigma^{2}\right)\right|$ when positive and from $-\left|N\left(\mu, \sigma^{2}\right)\right|$ when negative, with $\mu=1$ and $\sigma=0.1$. Second, to investigate the effect of the distribution of interaction strengths on community stability, we assumed that the population dynamics can be described by a generalized LotkaVolterra model (Neutel et al. 2002). Then, $J_{i j}=\gamma_{i j} x_{i}^{*}$ $(i \neq j)$ if species $i$ benefits from species $j$, and $J_{i j}=-\gamma_{i j} x_{i}^{*}$ otherwise, where $\gamma_{i j}$ is the interaction rate (i.e., preference sensu Staniczenko et al. [2013]) between species $i$ and $j$, and $x_{i}^{*}$ is the abundance of species $i$ at equilibrium. We inferred Jacobian off-diagonal coefficients by estimating interaction preferences and species abundances following the method of Staniczenko et al. (2013) (see also section (Quantifying interactions for different interaction types)).

\section{Null models to test interconnection patterns and their effects on stability}

To test whether the observed network exhibits a specific pattern in the way plants combine the mutualistic and the antagonistic networks, we considered a null model for both the binary network and the quantitative network: $\boldsymbol{T}_{\text {rand }}$ (or $q\left(\boldsymbol{T}_{\text {rand }}\right)$ ) for the quantitative network) were generated by separately swapping columns (i.e., plant species) in the original mutualistic and antagonistic partitions (1000 random column swaps in each $\boldsymbol{M}$ and $\boldsymbol{A}$ partition). The dimension (number of plants, herbivores, and pollinators) and the topology (including the distribution of preferences for the quantitative network) of the mutualistic and antagonistic networks were both preserved in randomized networks (see Appendix S2). When considering the Jacobian matrix $\mathbf{J}_{\text {rand }}$ for the quantitative networks, the null model also conserved the particular organization of the off-diagonal non-zero elements in each Jacobian sub-matrices corresponding to the mutualistic and antagonistic networks. This way, the null model generated networks differing from the observed one only by the way plants interconnect the two types of network. For both the binary and the quantitative networks, we generated 1000 randomized network matrices. For each random matrix, we evaluated interconnection patterns with our metrics (correlation of generalism and of similarity in partners) and calculated the stability $s$ of $\mathbf{J}_{\text {rand }}$. Then we compared metrics for the observed network to the distribution of these metrics for the randomized networks.

All analyses were performed with $\mathrm{R}$ 3.1.0 (R Development Core Team 2014) using the corpcor package for partial correlation and the GetPreferences function of Staniczenko et al. (2013). The source code to calculate stability $s$ from an empirical network is provided in Supplement 1. When testing a relationship between stability and network metrics, we used a Kendall's correlation coefficient, $\tau$, because the variables did not follow a normal distribution.

\section{RESULTS}

\section{How do plants connect pollination and herbivory networks?}

When considering the binary network, the correlation between plant mutualistic and antagonistic degrees in the observed data set was 0.28 . This value was higher than expected when the interconnection pattern between mutualistic and antagonistic network was randomized $(P=0.011$, Fig. 2a). This indicates that plants that tended to interact with many mutualistic partners tended to interact with many antagonistic partners as well. When considering the quantitative network, the correlation between mutualistic and antagonistic Shannon diversities of preferences was -0.011 in the observed data set and it was not different from expected with 

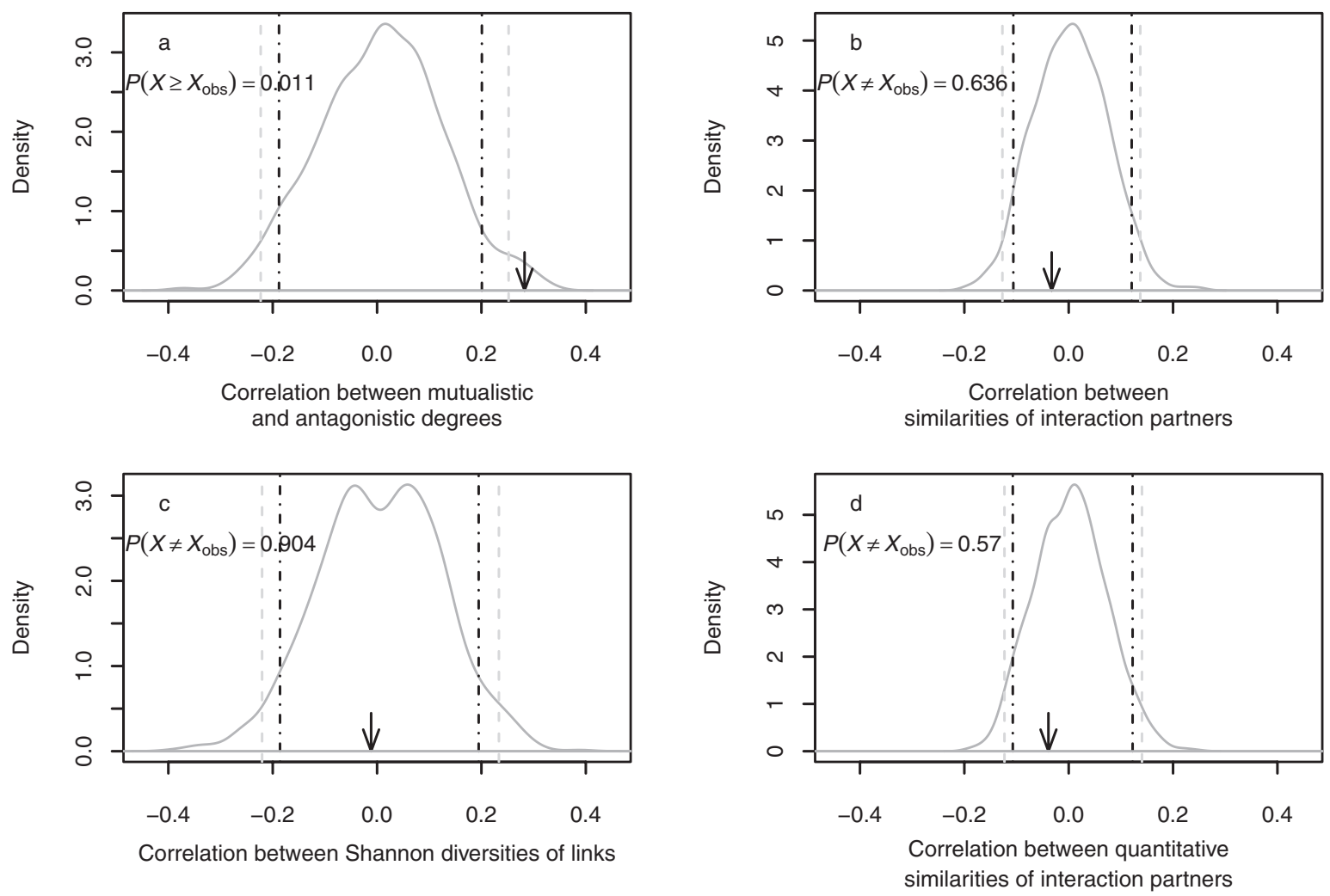

FIG. 2. Distribution of metrics of interconnection patterns in the observed and randomized networks. (a) The correlation between mutualistic and antagonistic degrees of plants, (b) the correlation between similarities of interaction partners, (c) the correlation between the mutualistic and antagonistic diversities of links, (d) the correlation between similarities of interaction partners when interactions are quantitative. The arrows indicate the observed values in the empirical network. The vertical gray dashed lines indicate the 2.5 th and 97.5 th percentiles. The vertical black dot-dashed lines indicate the 5 th and 95 th percentiles.

the null model $(P=0.904$, Fig. 2c). This indicates that there was no relationship between plants generalism in the mutualistic and the antagonistic networks when considering interaction preferences between the plants and the animals.

The correlation between similarities of interaction partners among plants in the binary network was -0.032 and was not significantly different from the random expectation of our null model $(P=0.636$, Fig. 2b). For the quantitative network, the correlation between similarities of interaction partners among plants was -0.038 and not significantly different from expected with the null model $(P=0.57$, Fig. $2 \mathrm{~d})$. Hence, plants that share the same pollinators did not necessarily share the same herbivores.

\section{Does the density of plants explain the observed pattern?}

We tested whether the abundance of a given species explained the observed positive correlation of degrees. The partial correlation between plant mutualistic and antagonistic degrees when LAI is held constant was still positive $(r(d \mathrm{M}, d \mathrm{~A}) . \mathrm{LAI}=0.14)$, but it did not differ significantly from 0 anymore $(P=0.200$, Fig. 3a).
The partial correlation between plant mutualistic and antagonistic degrees when number of floral units (FU) was held constant was $r(d \mathrm{M}, d \mathrm{~A}) . \mathrm{FU}=0.26$, and was significantly higher than $0(P=0.022$, Fig. $3 b)$. Hence, the LAI of plant species may explain most of the positive correlation between the two types of degree of plants: species with high LAI interacted with more species of pollinators and herbivores (Fig. 3a). Meanwhile, the number of floral units did not explain all the positive correlation between plant mutualistic and antagonistic degrees.

\section{Does the way plants combine pollination and herbivory networks contribute to stability?}

When comparing the eigenvalues of the community matrix of the binary data set to the ones obtained after randomization of the topology of the interconnected binary networks, we found that $s$ in the observed network was lower than expected with random interconnection topology $(P=0.004$, Fig. 4a). The observed binary network was thus more stable than expected. This result was robust to the consideration of quantitative interactions $(P=0.008$, Fig. 4b). 

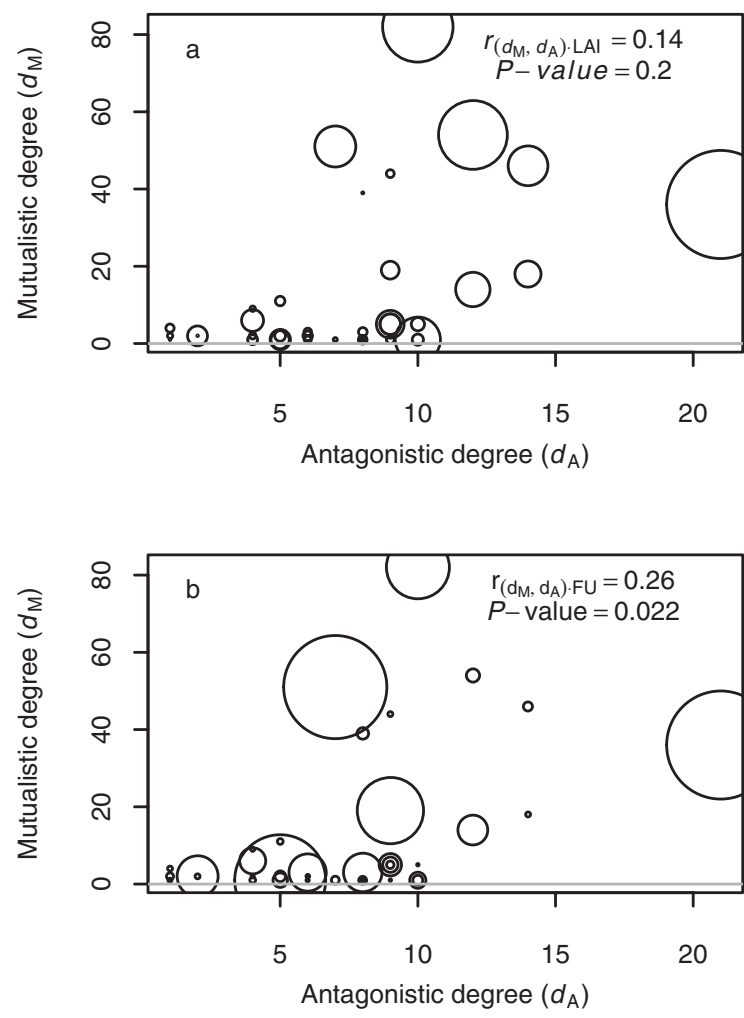

FIG. 3. The mutualistic degree $d_{\mathrm{M}}$ against the antagonistic degree $d$ of each plant. Each point is a plant species involved both in the pollination and the herbivory networks. The radius of the circle is proportional to (a) the leaf area index (LAI) or (b) the number of floral units (FU) of the plant species considered across the whole farm. The metrics $r_{(d \mathrm{M}, d \mathrm{~A}) . \mathrm{LAI}}$ and $r_{(d \mathrm{M}, d \mathrm{~A}) . \mathrm{FU}}$ are the partial correlations (with Kendall's method) between the mutualistic and the antagonistic degrees of plants, respectively LAI and FU being held constant. $P$ is the probability of getting such values when the mutualistic and antagonistic degrees are independent, associated with these tests.

Hence, the way plants combine pollination and herbivory networks in the observed data set appeared to promote stability.

To investigate which particular pattern of interconnection has a stabilizing effect, we looked for relationships between the two metrics of interconnection pattern and the stability metric $s$ in the randomized networks. For the binary network, we found a negative relationship between the stability metric $s$ and the correlation in plant mutualistic and antagonistic degrees $\left(\tau=-0.278, Z=-13.1642, P<2.2 \times 10^{-16}\right)$. This indicates that high correlation between plant mutualistic and antagonistic degrees might promote community stability. We also found a negative relationship between the stability metric $s$ for binary networks and the correlation between similarities of interaction partners of plants $(\tau=-0.246, Z=-11.6593, P<2.2 \times$ $10^{-16}$ ), suggesting that when plants tend to share both the same pollinators and herbivores the community was more stable. For the quantitative network, the stability metric was correlated neither with the
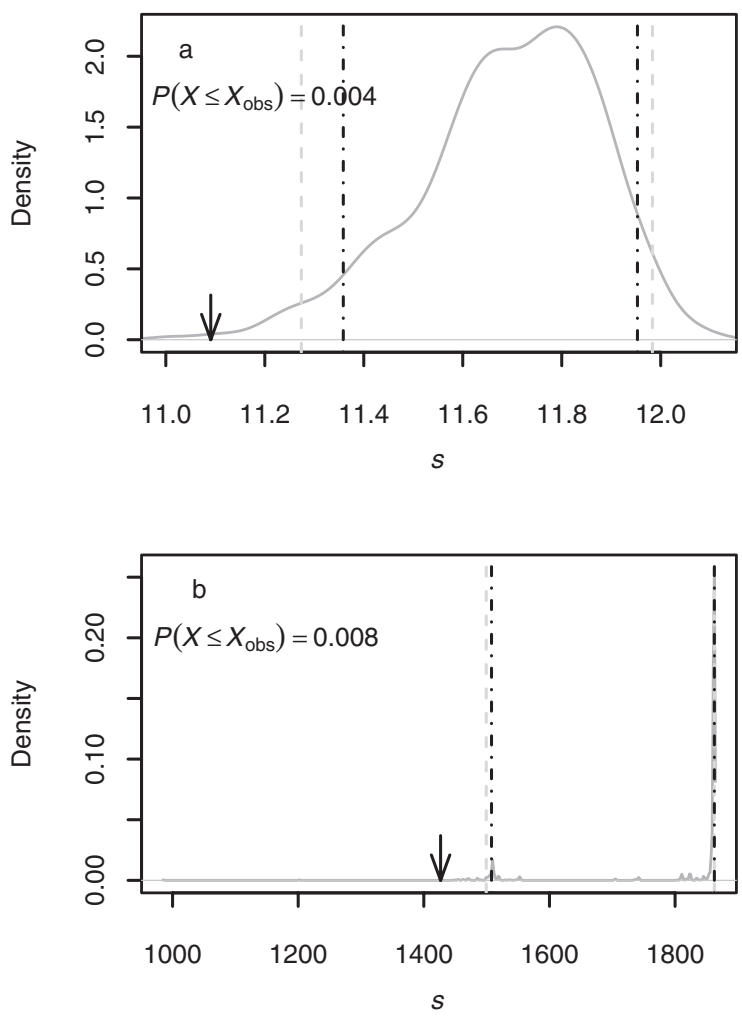

FIG. 4. Distribution of the stability $(s)$ of the observed and randomized networks (a) for binary networks and (b) when quantifying interaction strengths. The arrows indicate the observed values in the empirical network. The vertical gray dashed lines indicate the 2.5th and 97.5th percentiles. The vertical black dot-dashed lines indicate the 5th and 95th percentiles. The values lying on the right-hand side of the $x$-axis correspond to a lower stability.

correlation between Shannon diversities of preferences $(\tau=-0.015, Z=-0.7269, P=0.4673$ ) nor with the correlation between generalized similarities of interaction partners $(\tau=-0.007, Z=-0.3135, P=0.7539$ ). However, it was correlated with the correlation in plant mutualistic and antagonistic degrees $(\tau=-0.047$, $Z=-2.23, P=0.02575$ ), but not with the correlation between similarities of interaction partners for plants $(\tau=-0.002, Z=-0.1055, P=0.916)$.

\section{Discussion}

Our study showed that, for the Norwood farm data set (Pocock et al. 2012a,b), the way plants connect pollination and herbivory networks mattered for community stability, with the empirical pattern being more stable than random ones. When looking for the particular structures conferring stability, we found contrasting results depending on whether we considered the binary or the quantitative version of the network. For the binary network, both the investigated metrics were related to community stability and the empirical network was significantly different to random 
expectation for one of these metrics, i.e., the correlation between plants degrees (for mutualistic and antagonistic networks) was positive and appeared to promote stability. For the quantitative network, the two interconnected patterns were not associated with community stability and the metrics for the observed network were not significantly different from random expectations. Hereafter, we discuss these results in relation to previous research on plant traits, phylogenetic signal in interaction networks, and on the stability of ecological communities.

\section{How plants combine pollination and herbivory networks}

Our study of the Norwood farm network showed that plants that tend to interact with many pollinator species interacted with many herbivore species as well. Such a pattern appeared to be related to the differences in abundances among plant species. For the quantitative network, the community did not exhibit a positive correlation between plant generalism in herbivory and pollination networks anymore. Such discrepancy with the binary case might be explained by the use of preferences to quantify interaction as they "correct" for the effect of plants abundance. The importance of variation in abundances has previously been discussed for nestedness, where the explanation of the pattern by species abundances can be understood as a trivial outcome of variation in abundance (Blüthgen 2010, see also Staniczenko et al. 2013). Indeed, assuming random encounters among individuals (Vazquez and Aizen 2004), abundant plants are expected to interact with more pollinator and herbivore species. Nonetheless, abundance distributions and its explanatory power for network patterns can be seen as an important property of food webs that remains to be explored (Bascompte and Jordano 2007).

We expected that plants that share the same pollinators would share the same herbivores because in both plant-herbivore and plant-pollinator networks, it has been shown that closely related plant species are more likely to share the same herbivore species (Ives and Godfray 2006, Elias et al. 2013) and pollinator species (Rezende et al. 2007, Rafferty and Ives 2013). However, interestingly we did not find such a pattern in the network considered here and further analyses showed that there was no significant phylogenetic signal in the pollination network or in the herbivory network (see Appendix S3). This could explain the absence of an observed correlation between plant similarities of interaction partners. Low phylogenetic signal in pollination networks is often observed and is suggested to be due to convergences in the floral traits related to the attraction of particular pollinator taxa (Fernster et al. 2004, Rezende et al. 2007, Fontaine and Thébault 2015). However, plants as resources for herbivores are expected to exhibit a strong phylogenetic signal (Elias et al. 2013, Fontaine and Thébault 2015) and the absence of signal in our data set, even when performing the analysis for each type of herbivory separately, contrasts with these previous results (see Appendix S3).

\section{The way plants combine pollination and herbivory networks contributes to community stability}

We showed that the patterns of interconnection between pollination and herbivory networks were related to community stability, with the observed network being more stable than networks with randomized interconnections. Our findings are thus coherent with the numerous studies showing that the non-random structure of ecological networks such as food webs or mutualistic networks is key to their stability (e.g., Fox 2006, Okuyama and Holland 2008), and extend these previous results to the case of multi-interaction types. Our results thus join pioneering theoretical studies suggesting that the dynamics of mutualistic and antagonistic communities are dependent on each other (Ringel et al. 1996, Jang 2002, Bronstein et al. 2003) and that the way plants connect the two networks is an important structural component of ecological networks affecting the spread of perturbations (Melián et al. 2009, Fontaine et al. 2011, Sauve et al. 2014). As many previous studies, our approach of stability is subject to debate as it assumes a feasible equilibrium point (Rohr et al. 2014). The value of stability $s$ is not meaningful alone and should be treated with care. However, it is suitable for comparing random networks as we did here, and thus allows discussion on how interconnection patterns may affect community stability.

By connecting the pollination and herbivory networks, plants may undergo and channel both positive indirect effects mediated by pollinators (Ghazoul 2006, Carvalheiro et al. 2014), and negative indirect effects mediated either by herbivores or competing pollinators (Grover and Holt 1998, Chaneton and Bonsall 2000, Mitchell et al. 2009). A recent model for two plants sharing a pollinator and an herbivore showed that when mutualistic and antagonistic interactions have similar strengths, these indirect effects are theoretically expected to cancel each other, thus promoting stability (Sauve et al. 2015). The same mechanisms might explain the relationships between stability and the two studied interconnection patterns in the binary network case. Indeed, positive and negative indirect effects between plants are likely to cancel each other when the correlation between plant degrees in the two network types is positive and when plants that share the same pollinators also share the same herbivores. However, the distribution of interaction strengths strongly determines the nature of the resulting indirect interactions between the plants, leading to a wide range of possible indirect interactions (i.e., apparent facilitation, competition and even apparent antagonism) with complex consequences on community stability (Sauve et al. 2015). This might explain why we did not observe significant relationships between our measures of interconnection patterns 
and stability in the quantitative case. The higher stability of the empirical interconnection pattern combined with the limited effect of the interconnection patterns that we investigated, stress the fact that other interconnection patterns remain to be investigated, especially when dealing with quantitative interactions.

\section{Finding a common currency for different types of interactions}

Finally, although taking into account interaction strengths is clearly needed (e.g., Tang et al. 2014), our analysis highlights that considering different types of interactions together in a quantitative system raises the problem of defining a common currency to estimate interaction strengths (Fontaine et al. 2011). The frameworks commonly used to estimate interaction strengths in food webs cannot always be successfully applied to mutualistic systems (Vazquez et al. 2015): interaction frequencies are commonly taken as surrogate of interaction strength because they are quite straightforward to evaluate on the field (Vazquez et al. 2005, but see Vazquez et al. [2015] for a recent framework) but they can vary strongly between interaction types or organisms without being directly related to the actual magnitude of the effect on species growth rates. Consequently, the interaction types with highest frequencies may obscure the contribution of other interaction types to network patterns and community dynamics. To avoid these pitfalls in the present study, we chose to quantify the network interactions with preferences rather than interaction frequencies (Staniczenko et al. 2013). However, a preference value describes how likely an interaction between two individuals of two different species is to occur but not how much they affect plant growth. Thus, we assumed all interaction events to have the same effect in term of magnitude whatever the type of interaction. These assumptions are likely to affect the results and future studies should consider how the choice of the estimation of interaction strength could change our understanding of networks combining different types of interactions. Overall, our study stresses the need for the development of new metrics of network structure able to consider diverse types of interactions together. A better evaluation of the costs and benefits resulting from different interactions would also improve the way we integrate them in a single framework.

\section{Conclusion}

Our study is one of the first steps in the structural analysis of plant-pollinator-herbivore communities and puts forward interconnection patterns, a previously ignored aspect of network architecture (but see Melián et al. 2009). Our results clearly argue for a need for more empirical data sets combining various interaction types to further our understanding of the architecture and dynamics of such networks. A complementary approach to connect different ecological networks in a fruitful way could be to focus on species traits. Indeed, identifying plant traits that are relevant for different types of interactions, for example, either attracting or repelling both antagonists and mutualists of plants (Strauss 1997, Lehtilä and Strauss 1999, Strauss et al. 2002, Adler and Bronstein 2004, Adler et al. 2006, Theis 2006), could shed light on evolutionary processes such as conflicting selection (Strauss and Irwin 2004, Whittall and Strauss 2006), which are key in shaping the architecture of networks combining different interaction types. Overall, our study unveils an additional and understudied level of complexity of the architecture of biodiversity, and paves the way for an understanding of the web of life.

\section{ACKNOWLEDGMENTS}

We are grateful to Neil Williams and two anonymous reviewers for their constructive feedbacks on our manuscript. A. M. C. Sauve was supported with a fellowship by the Chaire "Modélisation Mathématique et Biodiversité" of Veolia Environnement - École Polytechnique - Museum National d'Histoire Naturelle - Fondation X.

\section{Literature Cited}

Adler, L. S., and J. L. Bronstein. 2004. Attracting antagonists: does floral nectar increase leaf herbivory? Ecology 85:1519-1526.

Adler, L. S., M. Wink, M. Distl, and A. J. Lentz. 2006. Leaf herbivory and nutrients increase nectar alkaloids. Ecology Letters 9:960-967.

Allesina, S., and S. Tang. 2012. Stability criteria for complex ecosystems. Nature 483:205-208.

Bascompte, J., and P. Jordano. 2007. Plant-animal mutualistic networks: the architecture of biodiversity. Annual Review of Ecology, Evolution and Systematics 38:567-593.

Bascompte, J., P. Jordano, C. J. Melián, and J. M. Olesen. 2003. The nested assembly of plant-animal mutualistic networks. Proceedings of the National Academy of Sciences USA 100:9383-9387.

Bersier, L.-F., C. Banasek-Richter, and M.-F. Cattin. 2002. Quantitative descriptors of food-web matrices. Ecology 83:2394-2407.

Blüthgen, N. 2010. Why network analysis is often disconnected from community ecology: a critique and an ecologist's guide. Basic and Applied Ecology 11:185-195.

Blüthgen, N., J. Fründ, D. P. Vázquez, and F. Menzel. 2008. What do interaction network metrics tell us about specialization and biological traits? Ecology 89:3387-3399.

Bronstein, J. L., W. G. Wilson, and W. F. Morris. 2003. Ecological dynamics of mutualist/antagonist communities. American Naturalist 162:S24-S39.

Carvalheiro, L. G., et al. 2014. The potential for indirect effects between co-flowering plants via shared pollinators depends on resource abundance, accessibility and relatedness. Ecology Letters 17:1389-1399.

Chaneton, E. J., and M. B. Bonsall. 2000. Enemy-mediated apparent competition: empirical patterns and the evidence. Oikos 88:380-394.

Dicks, L. V., S. A. Corbet, and R. F. Pywell. 2002. Compartmentalization in plant-insect flower visitor webs. Journal of Animal Ecology 71:32-43.

Elias, M., C. Fontaine, and F. J. F. van Veen. 2013. Evolutionary history and ecological processes shape a local multilevel antagonistic network. Current Biology 23:1355-1359. 
Evans, D. M., PocockM. J. O. , Brooks J, and Memmott J.. 2015. Abundance of plant taxa on Norwood Farm, Somerset during 2007 and 2008. NERC Environmental Information Data Centre. doi: doi:10.5285/0c123d4e-186f-48f5-8580-d0696b247287.

Fernster, C. B., W. S. Armbruster, P. Wilson, M. R. Dudash, and J. D. Thomson. 2004. Pollination syndromes and floral specialization. Annual Review of Ecology, Evolution, and Systematics 35:375-403.

Fontaine, C., and E. Thébault. 2015. Comparing the conservatism of ecological interactions in plant-pollinator and plantherbivore networks. Population Ecology 57:29-36.

Fontaine, C., P. R. Guimarães, S. Kéfi, N. Loeuille, J. Memmott, W. H. van der Putten, F. J. F. van Veen, and E. Thébault. 2011. The ecological and evolutionary implications of merging different types of networks. Ecology Letters 14:1170-1181.

Fox, J. W. 2006. Current food web models cannot explain the overall topological structure of observed food webs. Oikos 115:97-109.

Genini, J., L. P. C. Morellato, P. R. Guimarães, and J. M. Olesen. 2010. Cheaters in mutualism networks. Biology Letters 6:494-497.

Ghazoul, J. 2006. Floral diversity and the facilitation of pollination. Journal of Ecology 94:295-304.

Grover, J. P., and R. D. Holt. 1998. Disentangling resource and apparent competition: realistic models for plant-herbivore communities. Journal of Theoretical Biology 191:353-376.

Ings, T. C., et al. 2009. Ecological networks-beyond food webs. Journal of Animal Ecology 78:253-269.

Ives, A. R., and H. C. J. Godfray. 2006. Phylogenetic analysis of trophic associations. American Naturalist 168:E1-E14.

Jang, J. 2002. Dynamics of herbivore-plant-pollinator models. Journal of Mathematical Biology 44:129-149.

Kéfi, S., et al. 2012. More than a meal... integrating non-feeding interactions into food webs. Ecology Letters 15:291-300.

Kéfi, S., E. L. Berlow, E. A. Wieters, L. N. Joppa, S. A. Wood, U. Brose, and S. A. Navarrete. 2015. Network structure beyond food webs: mapping non-trophic and tropic interactions on Chilean rocky shores. Ecology 96:291-303.

Lehtilä, K., and S. Y. Strauss. 1999. Effects of foliar herbivory on male and female reproductive traits of wild radish, Raphanus raphanistrum. Ecology 80:116-124.

Lewinsohn, T. M., P. I. Prado, and P. Jordano. 2006. Structure in plant animal interaction assemblages. Oikos 113:174-184

Maron, J. L., and E. Crone. 2006. Herbivory: effects on plant abundance, distribution and population growth. Proceedings of the Royal Society B 273:2575-2584.

May, R. M. 1972. Will a large complex system be stable? Nature 238:413-414.

McCall, A. C., and R. E. Irwin. 2006. Florivory: the intersection of pollination and herbivory. Ecology Letters 9:1351-1365.

Melián, C. J., J. Bascompte, P. Jordano, and V. Krivan. 2009. Diversity in a complex ecological network with two interaction types. Oikos 118:122-130.

Memmott, J. 1999. The structure of a plant-pollinator food web. Ecology Letters 2:276-280.

Mitchell, R. J., R. J. Flanagan, B. J. Brown, N. M. Waser, and J. D. Karron. 2009. New frontiers in competition for pollination. Annals of Botany 103:1403-1413.

Neutel, A. M., J. A. P. Heesterbeek, and P. C. de Ruiter. 2002. Stability in real food webs: weak links in long loops. Science 296:1120-1123

Neutel, A.-M., J. A. P. Heesterbeek, J. van de Koppel, G. Hoenderboom, A. Vos, C. Kaldeway, F. Berendse, and P. C. de Ruiter. 2007. Reconciling complexity with stability in naturally assembling food webs. Nature 449:599-602.

Okuyama, T., and N. J. Holland. 2008. Network structural properties mediate the stability of mutualistic communities. Ecology Letters 11:208-216.
Ollerton, J., R. Winfree, and S. Tarrant. 2011. How many flowering plants are pollinated by animals? Oikos 120:321-326.

Pocock, M. J. O., D. M. Evans, and J. Memmott. 2010. The impact of farm management on species-specific leaf area index (LAI): farm-scale data and predictive models. Agriculture, Ecosystems and Environment 135:279-287.

Pocock, M. J. O., D. M. Evans, and J. Memmott. 2012a. The robustness and restoration of a network of ecological networks. Science 335:973-977.

Pocock, M. J. O., D. M. Evans, and J. Memmott. 2012b. Data from: the robustness and restoration of a network of ecological networks. Dryad Digital Repository: doi: 10.5061/ dryad.3s36r118.

R Development Core Team. 2014. R: A language and environment for statistical computing. R Foundation for Statistical Computing, Vienna, Austria.

Rafferty, N. E., and A. R. Ives. 2013. Phylogenetic trait-based analyses of ecological networks. Ecology 94:2321-2333.

Rezende, E. L., J. E. Lavabre, P. R. Guimarães, P. Jordano, and J. Bascompte. 2007. Non-random coextinctions in phylogenetically structured mutualistic networks. Nature 448:925-928.

Ringel, M. S., H. H. Hu, and G. Anderson. 1996. The stability and persistence of mutualisms embedded in community interactions. Theoretical Population Biology 50:281-297

Rohr, R. P., S. Saavedra, and J. Bascompte. 2014. On the structural stability of mutualistic systems. Science 345:416.

Sauve, A. M. C., C. Fontaine, and E. Thébault. 2014. Structurestability relationships in networks combining mutualistic and antagonistic interactions. Oikos 123:378-384.

Sauve, A. M. C., C. Fontaine, and E. Thébault. 2015. Stability of a diamond-shaped module with multiple interaction types. Theoretical Ecology 1-11.

Staniczenko, P. P. A., J. C. Kopp, and S. Allesina. 2013. The ghost of nestedness in ecological networks. Nature Communications 4:1391.

Strauss, S. Y. 1997. Floral characters link herbivores, pollinators, and plant fitness. Ecology 78:1640-1645.

Strauss, S. Y., and R. E. Irwin. 2004. Ecological and evolutionary consequences of multispecies plant-animal interactions. Annual Review of Ecology, Evolution and Systematics 35:435-466.

Strauss, S. Y., J. A. Rudgers, J. A. Lau, and R. E. Irwin. 2002. Direct and ecological costs of resistance to herbivory. Trends in Ecology and Evolution 17:278-285.

Szell, M., R. Lambiotte, and S. Thurner. 2010. Multirelational organization of large-scale social networks in an online world. Proceedings of the National Academy of Sciences USA 107:13636-13641

Tang, S., S. Pawar, and S. Allesina. 2014. Correlation between interaction strengths drives stability in large ecological networks. Ecology Letters 17:1094-1100.

Thébault, E., and C. Fontaine. 2008. Does asymmetric specialization differ between mutualistic and trophic networks? Oikos 117:555-563.

Thébault, E., and C. Fontaine. 2010. Stability of ecological communities and the architecture of mutualistic and trophic networks. Science 329:853-856.

Theis, N. 2006. Fragrance of Canada thistle (Cirsium arvense) attracts both floral herbivores and pollinators. Journal of Chemical Ecology 32:917-927.

Tylianakis, J. M., E. Laliberté, A. Nielsen, and J. Bascompte. 2010. Conservation of species interaction networks. Biological Conservation 143:2270-2279.

Van Veen, F. J. F., C. B. Müller, J. K. Pell, and H. C. J. Godfray. 2008. Food web structure of three guilds of natural enemies: 
predators, parasitoids and pathogens of aphids. Journal of Animal Ecology 77:191-200.

Vazquez, D. P., and M. A. Aizen. 2004. Asymmetric specialization: a pervasive feature of plant-pollinator interactions. Ecology 85:1251-1257.

Vazquez, D. P., W. F. Morris, and P. Jordano. 2005. Interaction frequency as a surrogate for the total effect of animal mutualists on plants. Ecology Letters 8:1088-1094.
Vazquez, D. P., R. Ramos-Jiliberto, P. Urbani, and F. S. Valdovinos. 2015. A conceptual framework for studying the strength of plant-animal mutualistic interactions. Ecology Letters 18:385-400.

Whittall, J. B., and S. Y. Strauss. 2006. Non-pollinator agents of selection on floral traits. Pages 120-138 in L. D. Harder and S. C. H. Barrett, editors. Ecology and evolution of flowers. Oxford University Press, Oxford, UK.

\section{SUPPORTING INFORMATION}

Additional supporting information may be found in the online version of this article at http://onlinelibrary.wiley.com/ doi/10.1890/15-0132.1/suppinfo 